\title{
Estudio clínico-epidemiológico sobre el síndrome de ardor bucal
}

\author{
López Carriches C*, Martínez-González JMa**, Gómez Font R*** \\ Leco Berrocal I****, Donado Rodríguez M*****
}

\section{RESUMEN}

En el síndrome de ardor bucal el paciente refiere un dolor crónico que no suele acompañarse de lesiones mucosas u otros signos clínicos.

Aunque la lengua es la localización más importante, también afecta a los labios, el paladar y la mucosa yugal e incluso a la garganta y el suelo de la boca.

Con el propósito de analizar los factores epidemiológicos y determinar si este síndrome se asocia con alguna alteración oral o sistémica se realizó un estudio sobre 25 pacientes con síndrome de ardor bucal que acudieron al servicio de Cirugía de la Facultad de Odontología de la Universidad Complutense de Madrid.

Se observó que tiene un componente psicógeno importantísimo presentando la mayoría de los pacientes unas características comunes y se puede afirmar que ciertos medicamentos como los antidepresivos influyen en este cuadro.

Palabras clave: Síndrome de ardor bucal, glosodinia, estomatodinia.

\section{ABSTRACT}

In burning mouth syndrome, patient refers a chronic pain that is not usually accompanied by mucosal lesions or another clinic signs.

Although tongue is the most important location, lips, palate, yugal mucosa even throat and mouth floor can be affected, too.

On the purpose of analysing the epidemiological aspects and to decide if this syndrome is associated with oral or systemic alterations a study was carried out with 25 burning mouth syndrome patients that came to the Surgery Unit of School of Dentistry of University Complutense of Madrid.

A very important psychological component has been observed and most of the patients presented quite common characteristic and it can be affirmed that certain medicines like antidepressive take influence in this syndrome.

Key words: Burning mouth syndrome, glossodynia, stomatodynia.

* Profesora colaboradora (UCM) y asociada (UEM).

** Profesor titular de Cirugía Maxilofacial. UCM.

*** Profesor responsable de Cirugía Bucal. UEM.

**** Profesora colaboradora de Cirugía Bucal (UCM).

***** Catedrático de Patología Quirúrgica Oral y Maxilofacial.(UCM).

López Carriches D, Martínez-González JM, Gómez Font R, Leco Berrocal L, Donado Rodríguez M. Estudio clínicoepidemiológico sobre el síndrome de ardor bucal. Av. Odontoestomatol 2003; 19-4: 185-191 
El síndrome de ardor bucal se presenta como un dolor crónico orofacial que no suele acompañarse de lesiones mucosas u otros signos clínicos (1).

Aunque la lengua es la localización más importante, también afecta a los labios y el paladar (2) e incluso la garganta, el suelo de la boca y las mejillas (3). En algunas ocasiones afecta solo a la mucosa del labio inferior. Se cree que los factores desencadenantes y el tratamiento son los mismos que cuando afecta a la lengua (4).

Actualmente este síndrome se inscribe dentro del dolor psicógeno (5). Se le denomina también glosodinia, glosopirosis, disestesia oral, estomatodinia, estomatopirosis, síndrome de boca ardiente, etc.

La mayoría de los pacientes son mujeres en edad postmenopáusica $(2,6)$ siendo la edad media de 59 años (1).

Aunque hay pocos estudios epidemiológicos se cita una frecuencia del 2,6\% al 5,1\% (6).

Se da tanto en pacientes dentados como en desdentados, aunque en mayor proporción en estos últimos.

Cabe distinguir en la etiología de este proceso los problemas orales, las condiciones sistémicas y los factores psicológicos.

Entre las posibles causas etiológicas orales se ha manejado la alergia a las prótesis, alimentos, productos de belleza e incluso el mercurio de las obturaciones (7), la irritación mecánica por prótesis, los hábitos parafuncionales, la disfunción de la ATM y los procesos de galvanismo $(2,3,6,8)$. También se cita como causa una posible disfunción de glándulas salivales (9).

Se sugiere también como causa etiológica la candidiasis, las infecciones virales, la lengua geográfica, la enfermedad periodontal y las enfermedades vesículo-ampollosas.

En cuanto a las condiciones sistémicas, la menopausia puede ser un factor etiológico del síndrome de ardor bucal. El $80 \%$ de los pacientes son mujeres postmenopausicas (10).
La ingesta de medicamentos está íntimamente relacionada con este síndrome: Hay una gran cantidad de medicamentos que producen xerostomía como los antidepresivos, antihipertensivos, antihistamínicos, etc. $(3,6)$. Se deben tener también en cuenta los enjuagues bucales que producen irritación local.

En enfermos inmunodeprimidos puede presentarse ardor bucal (VIH).

También es muy importante descartar las deficiencias nutricionales, ya que se ha asociado siempre a déficits de hierro, vitaminas B1, B2, B6, B12 (anemia perniciosa), ácido fólico (hay ardor bucal y queilitis angular) y cinc.

También se ha asociado el ardor bucal con la diabetes mellitus (11).

A pesar de la gran cantidad de factores mencionados es idiopática en muchos pacientes (6).

La personalidad y los cambios de humor (ansiedad y depresión están totalmente aceptados como factores en el ardor bucal, hasta el punto de que se considera una enfermedad psicógena $(2,3)$. Estos pacientes suelen tener problemas familiares como hijos discapacitados o que han sufrido accidentes de tráfico, etc. (12).

Algunas características de su personalidad son: Emocionalmente reprimidos, aislados socialmente, con cancerofobia, tendencias neuróticas, etc. Aquellos casos rebeldes al tratamiento se dan en pacientes más inestables, aprensivos e hipocondriacos.

Rojo et al (13) también encuentran asociación entre este sídrome y la psicología del paciente. Afirman que aparecen primero la depresión y la ansiedad pero que los síntomas dan lugar a un agravamiento de esta depresión y esta ansiedad. El paciente además presenta obsesión y hostilidad.

Hay una relación significativa entre la ansiedad y la depresión y los hábitos parafuncionales (como frotar los dientes) en pacientes con síndrome de ardor bucal (14). 
La clínica es insidiosa para el paciente, éste refiere ardor bucal, dolor, escozor, xerostomía y problemas en la percepción del gusto, con un sabor amargo o metálico (15).

Los pacientes tienen además otros síntomas menopáusicos como dolor de cabeza, alteración del sueño, problemas de salud no específicos así como disminución de la líbido y cambios de humor (irritabilidad y depresión).

Es muy significativa la capacidad de sacar la lengua y enseñarla (16).

Las papilas foliaceas están discretamente hiperplásicas y enrojecidas y el resto de las papilas linguales están atróficas (16).

Los niveles de tolerancia al dolor están disminuidos en estos pacientes. Son factores agravantes la tensión, la fatiga y las comidas calientes y reducen el dolor dormir, comer y distraerse.

Por la gran cantidad de pacientes que demandan tratamiento por este motivo, así como por la insidiosa clínica y difícil manejo del enfermo creemos ampliamente justificado la realización del presente trabajo, para el cual nos marcamos como objetivos establecer los factores epidemiológicos de este cuadro y determinar si este síndrome se asocia con alguna alteración oral o sistémica.

\section{PACIENTES Y METODOLOGÍA}

El estudio se realizó sobre 25 pacientes con síndrome de ardor bucal que acudieron al servicio de Cirugía Bucal de la Facultad de Odontología de la UCM en demanda de tratamiento. El estudio tuvo una duración de dos años.

A todos los pacientes se les realizó un completo examen intraoral con una completa anamnesis, inspección y palpación rellenando una ficha clínica especialmente diseñada para este propósito. (Ver tablas 1 y 2).

Además del examen de la cavidad bucal se realizó al paciente una historia clínica de su estado de salud

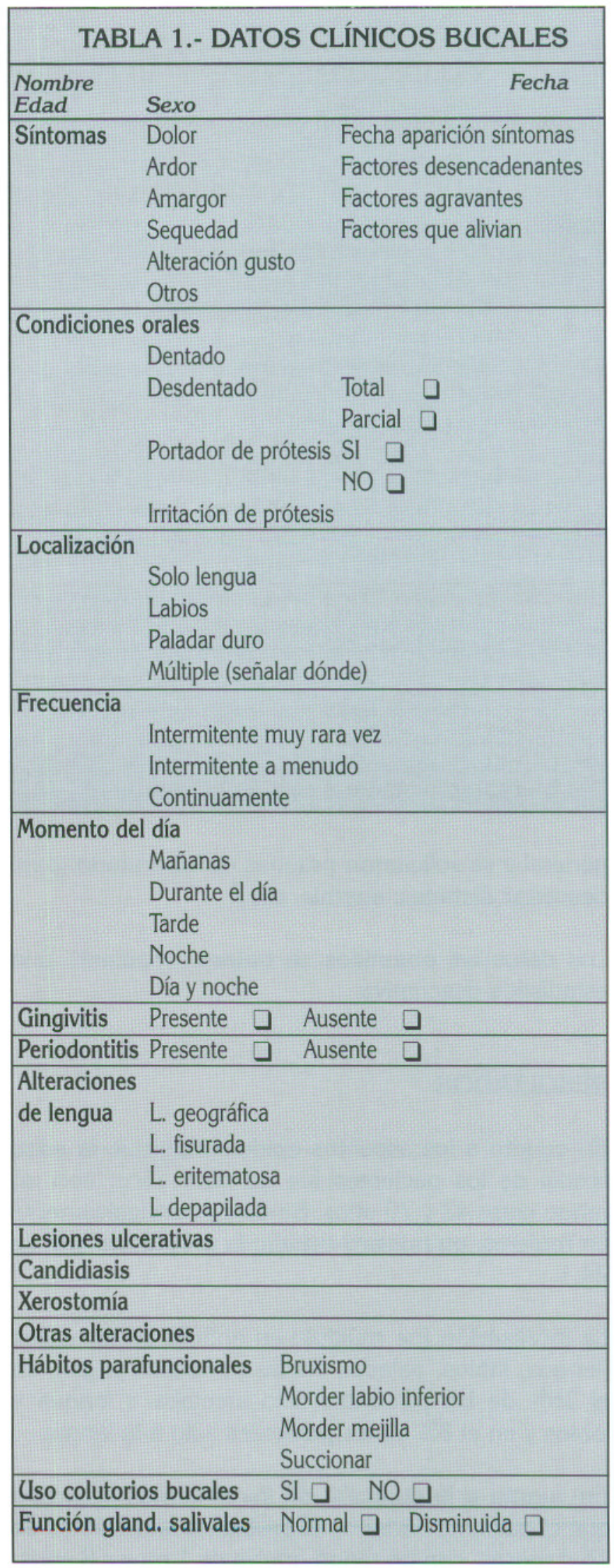




\begin{tabular}{|ll|}
\hline \multicolumn{2}{|c|}{ TABLA 2.- DATOS CLÍNICOS GENERALES. } \\
CONDICIONES SISTÉMICAS
\end{tabular}

general y se solicitaron pruebas de laboratorio (para descartar diabetes, anemia, etc.).

Los datos así obtenidos se trataron mediante una estadística descriptiva.

\section{RESULTADOS}

En cuanto a los aspectos epidemiológicos, la edad media de los pacientes fue de 60,3 años, con un rango entre 42 y 70 años. Se da casi exclusivamente en mujeres, en nuestro estudio la proporción fue de 24:1.

La localización fue múltiple en el $56 \%$ de los casos (lengua, labios, paladar, encías y mucosa yugal), en el $36 \%$ de los pacientes sólo afectaba a lengua y labios y en el $8 \%$ de los pacientes sólo a la lengua.

En cuanto a la duración de los síntomas o tiempo que el paciente lleva con el problema la media es de 15,4 meses, con un rango que va de 1 mes a 8 años.
Si se pregunta a los pacientes por los factores que desencadenaron el síndrome de ardor bucal 19 no lo podían achacar a ninguna causa, 3 a la colocación de prótesis dental, 1 al tratamiento odontológico sobre todo exodoncia y tartrectomía, otro paciente a los nervios y el último a una operación de amígdalas.

El 52\% de los pacientes considera que nada agrava el cuadro, mientras que un $40 \%$ siente que las comidas ácidas y/o picantes agravan los síntomas y un $8 \%$ ve aumentar las molestias por los nervios. Un $44 \%$ no siente alivio con nada, un $28 \%$ con caramelos o chicles, un $12 \%$ al realizar enjuagues, un $8 \%$ comer algo y otro $8 \%$ beber agua.

Un $80 \%$ de los pacientes son desdentados parciales, un $4 \%$ totales y un $16 \%$ dentados. De los desdentados el $52 \%$ son portadores de prótesis y de éstos el $24 \%$ afirman tener irritación por prótesis, mientras que el $76 \%$ afirma estar cómodo con ella.

En cuanto a la frecuencia de los síntomas el $80 \%$ los nota continuamente a lo largo del día, el $8 \%$ sólo por las mañanas y el $12 \%$ por la tarde. Los síntomas desaparecen durante el sueño.

Un $40 \%$ de los pacientes tiene gingivitis y un $16 \%$ periodontitis.

El $48 \%$ no presenta lesiones en la lengua y el resto presenta depapilación en el $20 \%$ de los casos, lengua fisurada en el $28 \%$ y con inflamación en las papilas foliaceas el $4 \%$. Ningún paciente presentaba lesiones ulcerosas en la mucosa bucal. Solo un paciente presentaba candidiasis.

El $48 \%$ tenía xerostomía apreciable clínicamente. Se han citado hábitos parafuncionales como causa de este síndrome: En nuestro estudio 9 pacientes tenían bruxismo (36\% de los casos).

Es significativo que el $88 \%$ de los pacientes estaba usando un colutorio bucal.

En cuanto a anomalías en la analítica solo un paciente era diabético.

Casi todos los paciente tomaban alguna medicación: 16 de ellos tomaban dos o más fármacos, 7 toma- 
ban un fármaco y sólo 2 no estaban tomando medicación en el momento de la consulta aunque los habían tomado en épocas anteriores. De todos los fármacos los mas frecuentes entre estos pacientes son los antidepresivos y /o ansiolíticos, utilizados por el $56 \%$ de los pacientes, los antihipertensivos eran usados por el $32 \%$, en menor proporción estaban los AINES (20\%), antiulcerosos y medicamentos del aparato digestivo (16\%), vitaminas (12\%) y suplementos tiroideos (8\%). Ya hemos señalado que varios pacientes tomaban más de un fármaco a la vez.

Todas las pacientes excepto una eran postmenopausicas. El 52\% de estos pacientes presentaban depresión tratada y un $96 \%$ reconoce tener ansiedad, 3 pacientes lloraron durante la historia clínica y reconocen tener cancerofobia. Un $40 \%$ afirma tener problemas graves en su vida personal (muerte de familiares, problemas con los hijos y/o con la pareja).

\section{DISCUSIÓN}

Según los resultados de nuestro estudio la edad media de presentación es de 60,3 años, lo que coincide con Klausner (1) que describe en su estudio una edad de 59 años. Coincidimos con todos los autores consultados en que es más frecuente en mujeres que en varones $(2,6)$.

La localización más importante es la lengua, aunque suele afectar a la vez a otras zonas de la mucosa oral, Lamey (4) describe casos de localización labial exclusivamente aunque en nuestro estudio no se ha dado este caso.

Estos pacientes cuando llegan a nosotros suelen haber visitado ya a múltiples profesionales y probado multitud de remedios. En cuanto a la etiología, la mayoría de los pacientes no saben a qué atribuirlo, algunos lo atribuyen a una visita a su dentista para la colocación de una prótesis, la realización de tartrectomía, etc., pero ninguno lo atribuye a la medicación que está tomando o a su estado de depresión o ansiedad. Como factores locales hemos podido constatar que el $24 \%$ de los pacientes presentaba irritación por prótesis y un 36\% de los pacientes era bruxista. Estos hallazgos coinciden con los de Maresky (6) quien afirma que los errores más fre- cuentes en estas prótesis son un aumento de la dimensión vertical, márgenes sobrextendidos y una oclusión incorrecta, aunque una prótesis mal realizada no es un desencadenante sino más bien un factor agravante. Sin embargo, Lamey (4) no ha encontrado ninguna relación con los hábitos parafuncionales. Nosotros creemos que el bruxismo no desencadena estos síntomas sino que es más bien un indicativo de que estas personas tienen un nivel de ansiedad mayor de lo normal, coincidiendo con las afirmaciones de Paterson (15) que utilizó una escala de ansiedad y depresión para demostrar una relación significativa con el bruxismo, en pacientes con ardor bucal.

En cuanto a una posible disfunción de glándulas salivales, aunque es cierto que el $48 \%$ de nuestros pacientes con ardor bucal presentaba xerostomía, hoy solo se admite que la conexión entre síndrome de ardor bucal y disfunción de las glándulas salivales es debida a problemas sistémicos y orales concurrentes y medicación; es decir, no hay diferencias en cuanto a función glandular entre pacienes con síndrome de ardor bucal y sujetos control. Se debe diferenciar muy bien la xerostomía real y la subjetiva pues la segunda tiene una base psicógena (6). Este síndrome tampoco está asociado a una disminución de las propiedades de protección y lubrificación de la saliva (concentración de proteinas, etc) (9).

En cuanto a los factores sistémicos tradicionalmente se ha asociado el ardor bucal con la diabetes mellitus, pero en realidad muy pocos paciente con glosodinia padecen diabetes. Cuando efectivamente la causa es la diabetes, se debe a neuropatías irreversibles y cambios en los vasos sanguíneos de los tejidos orales (2, $7,11)$. En nuestro estudio solamente un paciente era diabético y estaba bien controlado. Sin embargo sí parece tener una gran importancia la medicación que toma el paciente, y así hemos detallado en los resultados que la mayoría de ellos toma varios medicamentos, muchos de los cuales producen hiposialia o xerostomía como los antidepresivos o antihipertensivos. Se debe intertar sustituir estos fármacos por otros de la misma categoría con menor efecto de xerostomía y se debe procurar reducir el número de drogas que toma el paciente. El $88 \%$ de los pacientes estaba usando además un colutorio bucal que en muchas ocasiones no hace sino irritar la mucosa. 
Pero quizás son los factores psicológicos los que más peso tienen en este síndrome, lo que explicaría aquellos casos en los que no se dan ninguno de los factores arriba mencionados. En nuestro trabajo un 96\% de los pacientes reconoce tener ansiedad y muchos tienen o han tenido depresión. En otros estudios, al realizarles un cuestionario psicométrico, un número significativo de pacientes tenía problemas psiquiátricos, siendo la depresión la alteración más característica $(2,3)$. Según Kleinhanz (12) el dolor crónico y la depresión están muy relacionados: la depresión o trastorno psicológico aparecen antes que el ardor bucal. Llama la atención el hecho de que estos pacientes presentan unas características comunes como inestabilidad emocional (algunos lloran durante la historia clínica), problemas graves en su vida personal y como hemos dicho anteriormente ansiedad, en lo que coincidimos con Lamey (11), Rojo (13), Gorsky (17), etc.

El tratamiento de este tipo de pacientes es complejo, se debe eliminar la irritación por prótesis, cálculo,etc., y puede aliviar la aplicación tópica de glucocorticoides (acetato de hidrocortisona) aunque con precaución ya que tiene efectos secundarios como sequedad, etc.

Hay mucha controversia sobre los beneficios de una terapia hormonal con estrógenos. En el estudio realizado por Forabosco (18) en 1992 la mitad de los pacientes mostraron mejoría tras el tratamiento hormonal, aunque no se puede excluir un efecto placebo.

La aplicación de ácido cítrico como enjuague bucal o pilocarpina puede estimular el flujo salival mientras se toma la medicación antidepresiva. También puede ayudar mascar chicle y usar saliva artificial. Es importante mantener una correcta higiene bucal en estos pacientes y además se debe tranquilizar al paciente pues hemos observado que muchas veces sufre cancerofobia (3).

Actualmente se utiliza una amplia gama de fármacos para eliminar este problema: Analgésicos, sialogogos, reposición de vitaminas y minerales, tratamiento hormonal, benzodiacepinas, antidepresivos, antihistamínicos, corticosteroides, etc. $(19,20,21)$. Pero de todos ellos no se sabe cuál es realmente útil. Según Bergdahl $(7,22)$ sólo un tercio de los pacien- tes mejora con el tratamiento aplicado por no dar el tratamiento psicológico adecuado.

El uso de antidepresivos estaría justificado por el hecho de que el ardor bucal lleva asociado problemas psiquiátricos. En general, está demostrado que el uso de antidepresivos es útil en el tratamiento del dolor crónico no maligno cuando han fracasado otros tratamientos o cuando hay depresión asociada. Pero las dosis deben ser limitadas pues hay efectos anticolinérgicos que pueden agravar el cuadro (boca seca, visión borosa, etc.)

\section{CONCLUSIONES}

Aunque la relación con la mayoría de los factores etiológicos citados es especulativa, creemos que los factores psicológicos así como los efectos secundarios de ciertos medicamentos son muy importantes en la aparición y mantenimiento de este cuadro.

El tratamiento, muy complicado, se basa en una correcta historia clínica, tranquilizar al paciente, eliminar cualquier factor que pueda estar implicado (sarro, prótesis mal adaptadas...), administrar algún sialogogo o saliva artificial en casos de xerostomía asociada y reevaluar al paciente periódicamente.

\section{BIBLIOGRAFÍA}

1. Klausner J: Epidemiology of chronic facial pain: diagnostic usefulness in patient case. JADA 1994; 73: 567-71.

2. Ship J; Grushka M: Bourning mouth syndrome: An update. JADA 1995; 126: 843-52.

3. Tourne L et al: Bourning mouth syndrome. Oral Surg Oral Med Oral Pathol 1992; 74: 158-67.

4. Lamey PJ et al: Lip component of bourning mouth syndrome. Oral Surg Oral Med Oral Pathol 1994; 78: 590-3.

5. Van der Bijl P: Psycogenic pain in dentistry. Compendium 1995; 16: 46-53. 
6. Maresky LB; Van der Bijl P: Bourning mouth syndrome. Oral Surg Oral Med Oral Pathol 1993; 75: 303-7.

7. Bergdahl $\mathrm{J}$ et al: Cognitive therapy in the treatment of patients with burning mouth. Scan J Dent Res 1994; 102: 299-305.

8. Bergdahl M; Bergdahl J: Burning mouth syndrome: prevalence and associated factors. J Oral Pathol Med 1999; 28: 350-4.

9. Tammiala-Salonen $\mathrm{T}$ et al: Protein composition, adhesion and agglutination properties of saliva in burning mouth syndrome. Scan J Dent Res 1993; 101: 215-8.

10. Zachariasen RD: Oral manifestation of menopause. Compendium 1994; 14: 1584-90.

11. Lamey PJ et al: Type 3 burning mouth syndrome: psychological and allergic aspects. J Oral Pathol Med 1994; 23: 216-9.

12. Kleinhanz M; Bath R; Littner N: Antecedent of burning mouth syndrome (glossodynia). Recents life events vs psycopathologic aspect. J Dent Res 1994; 73: 567-71.

13. Rojo L et al: Prevalence of psychopatology in burning mouth syndrome. Oral Surg Oral Med Oral Pathol 1994; 78: 312-6.

14. Bergdahl $\mathrm{J}$ et al: Cognitive therapy in treatment of patients with resistant burning mouth syndrome: a controlled study. J Oral Pathol Med 1995; 24: 213-5.
15. Paterson AJ et al: Bourning mouth syndrome: The relationship between the HAD scale and parafuntional habits. J Oral Pathol Med 1995; 24 : 289-92.

16. Lucas Tomas M: Medicina Oral. Ed. Salvat. 1998.

17. Gorsky M et al: Clinical characteristics and management outcome in the bourning mouth syndrome. Oral Surg Oral Med Oral Pathol 1991; 72: 192-5.

18. Forabosco A et al: Efficacy of hormone replacement therapy in postmenopausal women with oral discomfort. Oral Surg Oral Med Oral Pathol 1992; 73: 570-4.

19. Sardella A; Uglietti D; Demarosi F; Lodi G; Bez C; Carrassi A: Benzydamine hydrochoride oral rinses in management of burning mouth syndrome. Oral Surg Oral Med Oral Pathol 1999; 88: 683686.

20. Grushka M; Epstein J; Mott A: An openlabel, dose scalation pilot study of the effect of clonazepan in burning mouth sydrome. Oral Surg Oral Med Oral Pathol 1998; 86 (5): 557-61.

21. Femiano F; Gombos F; ScullyC; BusciolanoM; De Luca P: Burning mouth syndrome (BMS): controlled open trial of the eficay of alpha-lipoic (trioctic acid) on symmptomatology. Oral Diseasses 2000; 6: 274-277.

22. Bergdahl J; Anneroth G: Bourning mouth syndrome: literature review and model for research and management. J Oral Pathol Med 1993; 22: 433-8. 